\title{
Preoperative Surgical Approach Planning for Metastatic Pituitary Stalk Tumor Using Multimodal Fusion Imaging in a Neuronavigation System -Case Report-
}

\author{
Shoko Merrit YAMADA, Noritaka MASAHIRA, Naoki IKAWA, \\ Eiichi NAKAI, Kae Chang PARK, and Keiji SHIMIZU \\ Department of Neurosurgery, Kochi Medical School, Kochi University, Nankoku, Kochi
}

\begin{abstract}
A 72-year-old woman presented with a metastatic brain tumor around the pituitary stalk compressing the chiasm anteriorly and upward. After tumor resection by a left pterional approach failed, a threedimensional (3D) image of the area of interest was reconstructed using the Vecter Vision ${ }^{2}$ navigation system and iPlan Cranial planning software version 2.5. Preoperative 3D computed tomography and magnetic resonance imaging data were fused to demonstrate the anatomical relationship between vessels, nerves, and tumor. Clearer demonstration of the optic nerves utilized thin slice axial and sagittal views along the nerves. The 3D reconstructed image demonstrated the spatial relationship of the tumor and surrounding tissue, and suggested the necessity of a right orbitozygomatic approach to create adequate working space for tumor resection. Second surgery according to this preoperative planning was successful. High quality multimodal fusion images in a navigation system has distinct advantages in preoperative assessment of essential structural relationships allowing adequate exposure of certain lesions and surrounding structures in individual patients by defining specific surgical approaches.
\end{abstract}

Key words: multimodal image fusion, planning software, neuronavigation, three-dimension

\section{Introduction}

Neuronavigation systems are increasingly used in neurosurgical procedures to provide real-time intraoperative image guidance. ${ }^{1,12,13)}$ In addition to the intraoperative use of the navigation system, the preoperative planning technique of multimodal image fusion of three-dimensional (3D) reconstruction of computed tomography (CT) and magnetic resonance (MR) imaging allows neurosurgeons to perform more accurate and less invasive operations. ${ }^{4,5}$ However, the practical application of $3 \mathrm{D}$ preoperative

Received April 6, 2009; Accepted July 27, 2009 planning images has not been compared to intraoperative views. The present study describes the utilization of this technique for preoperative decision-making in terms of the appropriate surgical approach to a brain tumor.

\section{Materials and Methods}

MR imaging used a Signa Horizon 1.5T (GE, Milwaukee, Wis., U.S.A.) with conditions as follows: $\mathrm{T}_{1}$-weighted imaging, fast spoiled gradient recalled scanning sequence, slice thickness of $1.5 \mathrm{~mm}$ with a slice gap of $0 \mathrm{~mm}$, echo time/repetition time $=2.8 / 12$ or $4.2 / 13 \mathrm{sec}$, matrix of 256 $\times 256$, and $240 \mathrm{~mm}$ field of view. Gadopentetate dimeglu- 
mine (Magnevist; Nihon Schering, Osaka) $(0.2 \mathrm{ml} / \mathrm{kg})$ was intravenously injected as contrast medium. Axial and sagittal $\mathrm{T}_{1}$-weighted images along each optic nerve (ON) were obtained for clearer visualization of the ONs and optic chiasm.

CT used an Aquilion TSX-101A (Toshiba Medical Systems, Tokyo) with conditions as follows: helical multi-slice scanning, $1.0 \mathrm{~mm}$ slice thickness, and helical pitch of 15.0. Iopamidol (Oypalomin300; Fuji Pharmaceuticals, Tokyo) was used as contrast medium mainly for vessel study. Iopamidol $(90 \mathrm{ml})$ was injected intravenously into the patient at $3 \mathrm{ml} / \mathrm{sec}$, and CT scanning was started $20 \mathrm{sec}-$ onds after the injection was initiated. The neuronavigation system was a Vector Vision ${ }^{2,12)}$ with iPlan Cranial planning software version 2.5 (Brain LAB, Feldkirchen, Germany). This software allows for 3D fusion of several images of MR imaging and CT.

iPlan Cranial is automatic image fusion software that does not require a clear anatomical reference point in each image for fusion, which is entirely different from a paired-point fusion system. DICOM (digital imaging and communication in medicine) data from CT and MR imaging are translated into gray scale values, and anatomical features of all intracranial tissues are three-dimensionally recognized as gray scale values. The automatic image fusion system identifies the gray scale value of each tissue, and fuses each image following the gray scale consistency. The fusion initiates from anatomically intelligible sections such as the rough facial surface, nasal cavity, and lateral ventricle, and the broadly fused images are gradually corrected by uniting more subtle structures. The fusion is finally accomplished when the software recognizes that the fused images are visually and logically consistent. The software reconstructs two different directional sequences from only one directional sequence, and synchronously restructures the three directional sequences as volume data. Therefore, the automatic image fusion software can adjust several different modalities of MR imaging and CT without relying on imaging parameters such as image slice angles, ranges, or thickness for fusion.

Six fiducial markers are affixed on the patient's head including the forehead, the pterions, and the asterions for more accurate navigation registration before CT and MR imaging. It is possible to perform navigation registration without the preoperative fiducial markers if a laser pointer is used in the operation room; however, we prefer to use fiducial markers for more precise registration. After the patient was positioned for surgery, the acquired data was matched to the patient's head position for registration.

\section{Case Presentation}

A 72-year-old woman, who had previously undergone removal of a right renal clear cell carcinoma, complained of rapidly progressing visual disturbance. MR imaging with gadopentetate dimeglumine revealed a well-defined enhanced lesion around the pituitary stalk, compressing the chiasm anteriorly and upward (Fig. 1). The patient had been receiving interferon alpha, and whole body radiological examinations identified no other metastasis. The

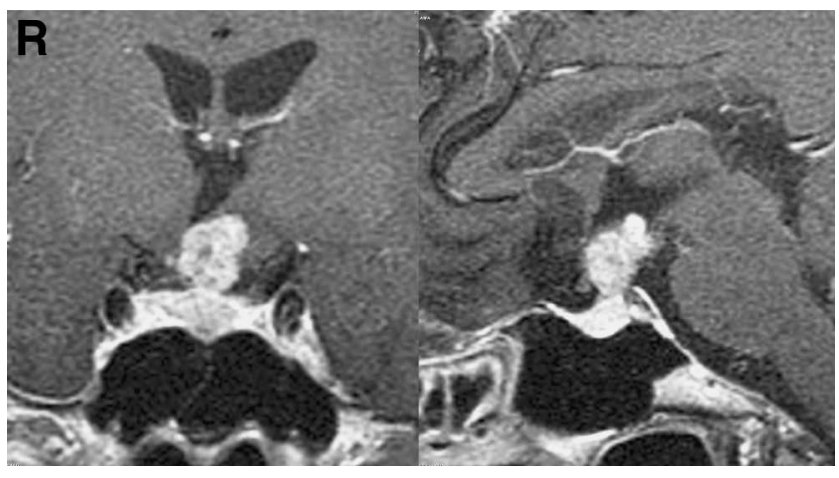

Fig. 1 Preoperative $T_{1}$-weighted magnetic resonance images with gadopentetate dimeglumine showing a mass in the pituitary stalk, compressing the chiasm anteriorly and upward. The tumor demonstrates left side dominance and adheres to the left hypothalamus.

patient was otherwise doing well. Surgical removal was considered to be the most appropriate treatment for this patient with progressive visual impairment.

Surgical approaches to the pituitary stalk usually include subfrontal, pterional, and trans-sphenoidal approaches as performed in removal of craniopharyngioma. The trans-sphenoidal approach was out of consideration because the pituitary gland was not involved in the tumor. Tumor resection was initially attempted through a left pterional approach due to the tumor location and adherence to the chiasm on the left. The navigation system was used only for real-time intraoperative image guidance. After separation of the left sylvian fissure, the left internal carotid artery (ICA), anterior cerebral artery, middle cerebral artery, and left $\mathrm{ON}$ were easily identified. The tumor was identified under the operating microscope in the space between the ICA and ON, as confirmed by the navigation system. However, the horizontal portion of the left anterior cerebral artery $\left(\mathrm{A}_{1}\right)$ was inflexible, so that movement of either the left ON upward or the left ICA laterally was difficult, which hampered the creation of adequate working space for tumor resection between the ICA and ON. Therefore, the surgery was completed without resecting any portions of the tumor.

To elucidate the cause of failure in the left pterional approach to the tumor and to clarify whether adequate working space for tumor resection could be obtained by a right side approach, iPlan Cranial planning software version 2.5 was used to fuse MR and CT images in a 3D format to clearly demonstrate the anatomical relationships of the arteries, ONs, optic chiasm, and tumor. To delineate the ON clearly, thin slice axial and sagittal MR imaging views were obtained along the left optic tract for postoperative analysis of the left side approach, and along the right optic tract for preoperative analysis of the right side approach. The anterior communicating artery was located at the right side of the chiasm very close to the right $\mathrm{ON}$, and the left $\mathrm{A}_{1}$ traveling from posterior to anterior was difficult to move laterally as noted intraoperatively (Fig. 2A). As seen in the operative view (Fig. $2 \mathrm{~B}$ left), the left $\mathrm{A}_{1}$ was stretch- 

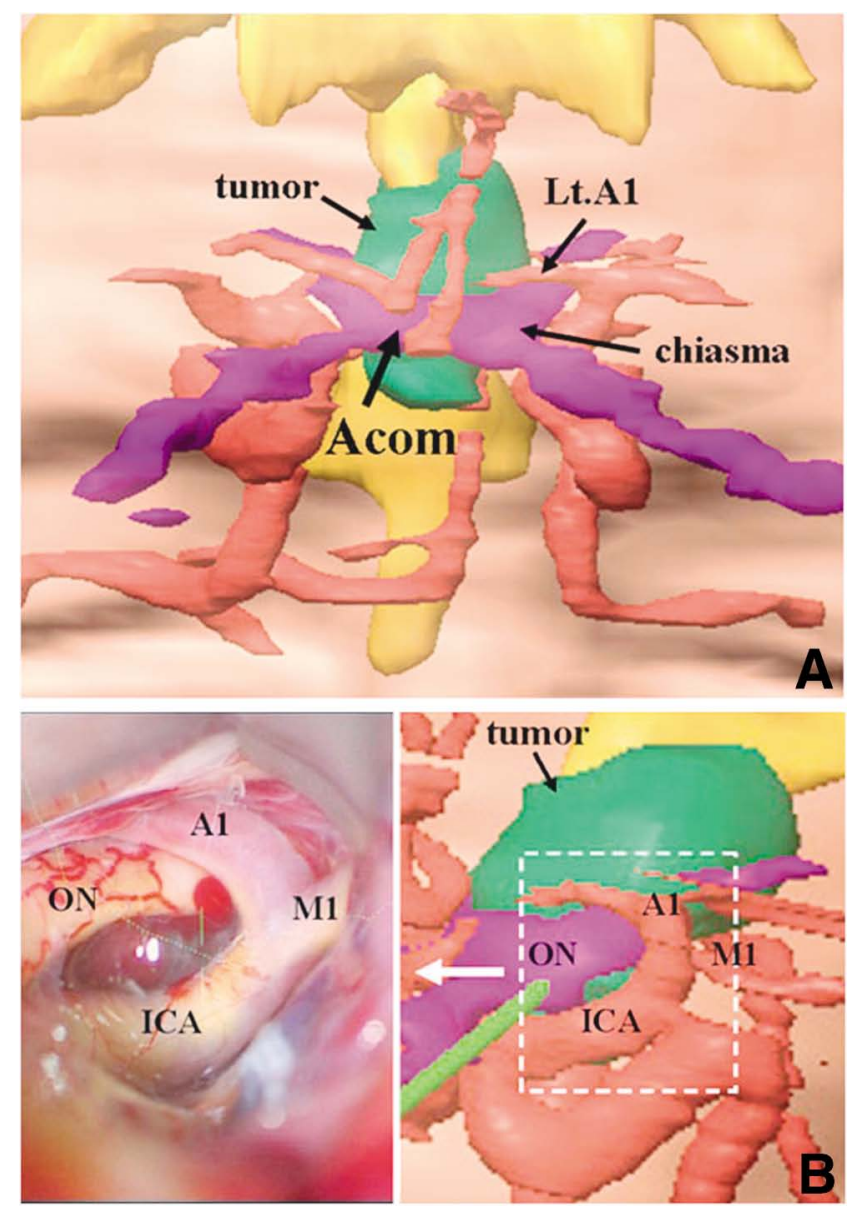

Fig. 2 A: Three-dimensional (3D) reconstructed image of the chiasm, arteries, and tumor showing the anterior communicating artery (Acom) is located above the right optic nerve and the left horizontal portion of the anterior cerebral artery (It $A_{1}$ ) and stretches from posterior to anterior. B: Intraoperative photograph of left internal carotid artery (ICA)- $A_{1}$-optic nerve (ON) triangle (left) showing the left $A_{1}$ runs just above the chiasm causing poor upward mobility of the artery. Corresponding 3D reconstructed image (right) showing a very narrow working space of the triangle with the horizontal portion of the middle cerebral artery $\left(M_{1}\right)$ and the area of the intraoperative view (white dotted square).

ed to the medial side running just above the left ON, and the top of the left ICA was adjacent to the chiasm, exactly as seen in the reconstructed imaging (Fig. 2B right). Therefore, the surgical space defined by the ICA, the $\mathrm{A}_{1}$, and the ON (ICA- $\mathrm{A}_{1}-\mathrm{ON}$ triangle) would be unduly restrictive due to the narrowness on the left side.

The next step was to estimate the working space within the right ICA- $\mathrm{A}_{1}-\mathrm{ON}$ triangle in the right side approach. The immovability of arteries, nerves, or tumor is not evaluated by the 3D fusion imaging, but the tip of the right ICA and the chiasm were separated by some distance, and the right $A_{1}$ formed a gentle arch separate from the ON, suggesting that the right ICA could be moved laterally and the right ON upward to obtain adequate space for tumor resection in the right ICA- $\mathrm{A}_{1}-\mathrm{ON}$ triangle as seen in the
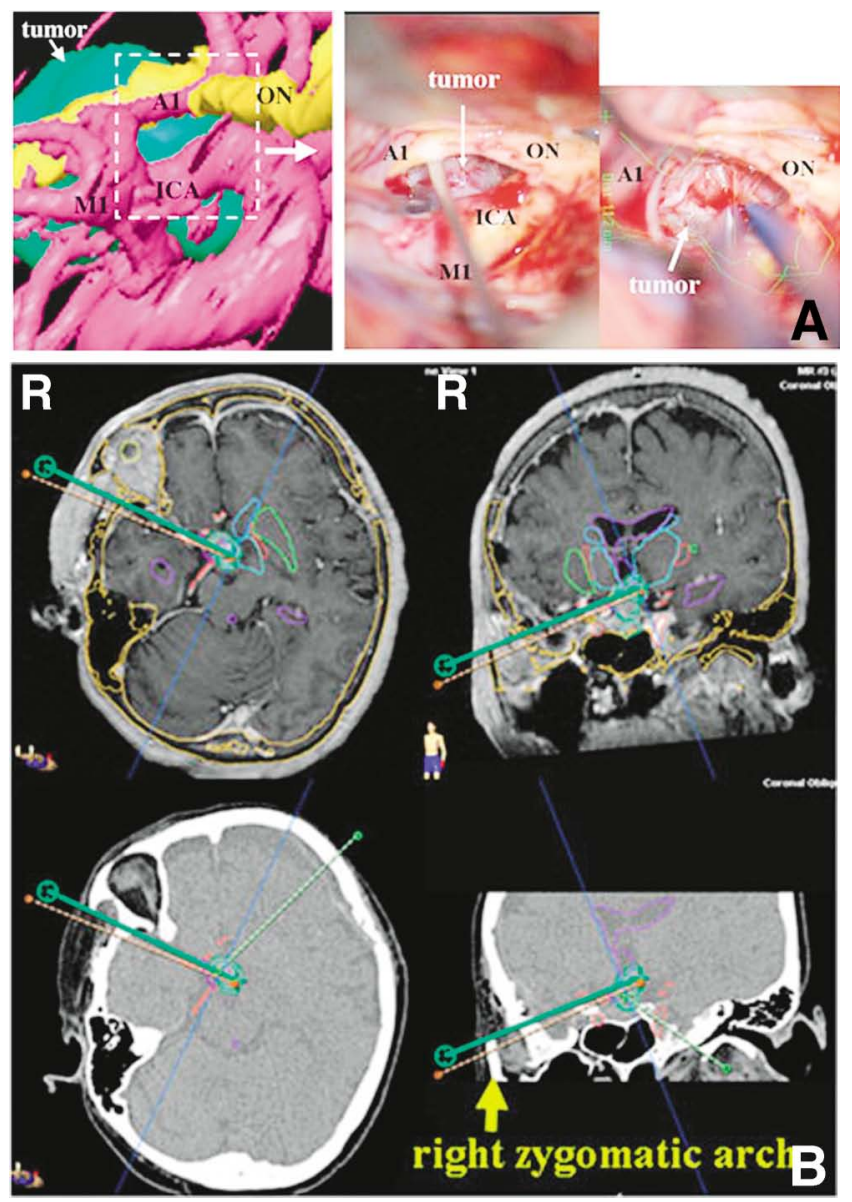

Fig. 3 A: Three-dimensional image of the right internal carotid artery (ICA)- $\mathrm{A}_{1}$-optic nerve $(\mathrm{ON})$ triangle showing much greater width because of elevation of the right $A_{1}$ (left). Therefore, an upward approach from the zygomatic arch to the tumor effectively utilizes the working space of the triangle. Intraoperative photographs (center and right) demonstrating the widely opened working space and the easily recognizable tumor. B: Preoperative planning of the surgical approach showing the necessity for the orbitozygomatic approach to reach the top of the tumor, with the area shown in the intraoperative view (white dotted square).

preoperative imaging (Fig. 3A left). The posterior portion of the pituitary stalk and the hypothalamus are generally difficult to recognize in the pterional approach. The preoperative planning imaging demonstrated the necessity to remove the right orbital roof and zygomatic arch to reach the top of the tumor attached to the hypothalamus (Fig. 3B). Therefore, we decided to perform a second operation using the right orbitozygomatic approach. As the preoperative 3D multimodal fusion imaging suggested, we actually obtained enough working space for the tumor resection by this approach (Fig. $3 \mathrm{~A}$ center and right). The right ICA and $A_{1}$ were easily moved laterally, and the tip of the tumor contacting the left hypothalamus was identified under the operating microscope in this approach. Therefore, the surgery was successfully completed based on the specific findings of preoperative anatomical recon- 


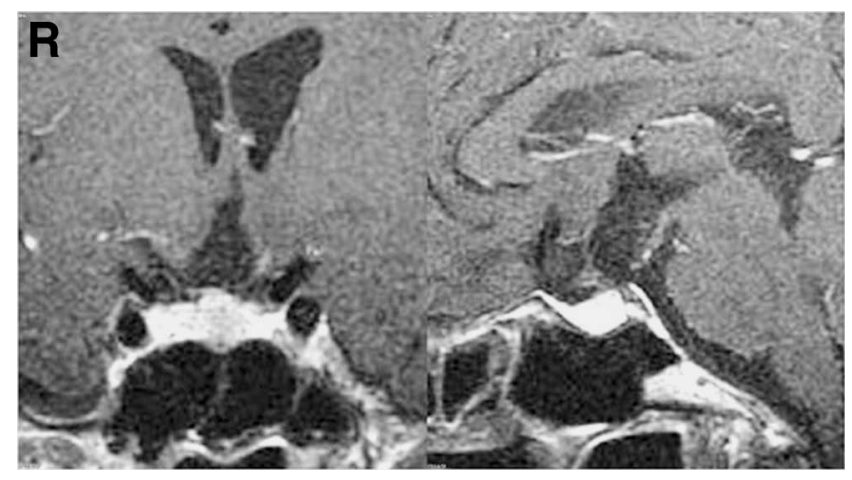

Fig. 4 Postoperative $T_{1}$-weighted magnetic resonance images with gadopentetate dimeglumine performed 2 weeks after the surgery revealing complete resection of the tumor.

struction of the lesion by 3D multimodal fusion imaging.

Postoperative MR imaging demonstrated complete resection of the tumor (Fig. 4), and the patient's visual acuity and visual fields recovered well after surgery. The patient was discharged one month after the surgery with mild diabetes insipidus which was well controlled by desmopressin medication.

\section{Discussion}

Renal clear cell carcinoma has been reported as radiation resistant. ${ }^{6)}$ Conversely, gamma knife surgery was effective for brain metastasis from renal cell carcinoma. ${ }^{8)}$ However, radiation therapy for tumor in contact with the ONs or optic chiasm could possibly induce optic neuropathy, as the radiation tolerance of the ONs and optic chiasm is approximately $10 \mathrm{~Gy},{ }^{11)}$ but hypofractionated cyberknife radiosurgery was recently reported to be effective for pituitary adenomas within $2 \mathrm{~mm}$ of the optic chiasm, without affecting the patients' vision. ${ }^{3)}$ Radiosurgery was considered in this case, but surgical removal was chosen because no other metastatic lesion was identified and the general condition of the patient was good enough to tolerate general anesthesia, the visual disturbance progressed rapidly necessitating early decompression of the optic chasm with immediate reduction of the tumor mass, no histological diagnosis of metastasis from renal cell carcinoma had been established, and there is no evidence that hypofractionated radiosurgery is effective for renal cell carcinoma metastasis to the pituitary stalk.

Neuronavigation systems have been used to determine the appropriate operative positioning for the patient, the degree of head rotation, and the extent of skin incision and craniotomy prior to surgery, in addition to intraoperatively confirming the depth and direction to the target point from a particular sulcus or gyrus. ${ }^{10)}$ These systems are also important for preoperative evaluation through multimodal image fusion software that demonstrates the spatial anatomical relationship of the blood vessels, the nervous system, and lesions. ${ }^{2,9)}$ In our case, the multimodal fusion image showed the anatomical relationships between the ON and arteries, which helped to clarify the reasons for failure in the first operation and to achieve success in the second surgery. The orbitozygomatic approach to the tumor was satisfactory because the left hypothalamus was easily recognized without exerting force on the $\mathrm{ON}$.

Current development of the fusion imaging technique allows fusion of several images of CT and MR imaging from any angle. Furthermore, the technological progression of imaging methods including MR imaging, CT, MR angiography, tractography, and scintigraphy will allow fusion of high quality images to visualize more clearly not only the precise anatomical 3D structure but also the functional areas that should not be surgically resected. Recently, tractography data of major white matter tracts were integrated into 3D datasets and applied in neuronavigation to provide visualization of the fiber tracts in the surgical field during resection of gliomas. ${ }^{7)}$ However, high quality images of each modality are required to obtain high quality multimodal fusion imaging because the quality of the final fusion image is dependent on the lowest quality image.

iPlan Cranial is automatic image fusion software, but artificial manipulation is necessary for computer recognition of individual tissues by delineating tumor, brain tissue, bone, nerves, and vessels differently. To acquire higher quality and more useful images, the targeted nerves must be visualized more clearly by obtaining thin slices along the nerves, because the vessels and tumors are relatively easy to reconstruct due to clear enhancement. In this case, we tried to demonstrate the bilateral ONs clearly using sagittal and axial views along each nerve. The $3 \mathrm{D}$ reconstructed imaging by high quality multimodal image fusion provided good visualization of the position of the lesion and surrounding structures, and was valuable in decision making regarding the specific surgical approach.

The preoperative planning of the surgical approach by iPlan Cranial contributed to the successful tumor resection in our case. However, reconstruction of high quality $3 \mathrm{D}$ images is not the ultimate solution and does not guarantee a good surgical outcome. With present radiological techniques, small arteries such as perforating arteries or tumor adhesion to surrounding tissue cannot be evaluated, which are both important factors in neurosurgical operations, so further improvement based on 3T MR imaging is expected to lead to more effective surgical outcomes.

\section{References}

1) Germano IM, Villalobos H, Silvers A, Post KD: Clinical use of the optical digitizer for intracranial neuronavigation. Neurosurgery 45: 261-270, 1999

2) Jung TY, Jung S, Kim IY, Park SJ, Kang SS, Kim SH, Lim SC: Application of neuronavigation system to brain tumor surgery with clinical experience of 420 cases. Minim Invasive Neurosurg 49: 210-215, 2006

3) Killory BD, Kresl JJ, Wait SD, Ponce FA, Porter R, White WL: Hypofractionated CyberKnife radiosurgery for perichiasmatic pituitary adenomas: early results. Neurosurgery 64(2 Suppl): A19-25, 2009

4) Kurtsoy A, Menku A, Tucer B, Oktem IS, Akdemir H: Neuronavigation in skull base tumors. Minim Invasive Neurosurg 48: 7-12, 2005 
5) Metzner R, Eisenmann U, Wirtz CR, Dickhaus H: Pre- and intraoperative processing and integration of various anatomical and functional data in neurosurgery. Stud Health Technol Inform 124: 989-994, 2006

6) Mori Y, Kondziolka D, Flickinger JC, Logan T, Lunsford LD: Stereotactic radiosurgery for brain metastasis from renal cell carcinoma. Cancer 83: 344-353, 1998

7) Nimsky C, Ganslandt O, Merhof D, Sorensen AG, Fahlbusch $\mathrm{R}$ : Intraoperative visualization of the pyramidal tract by diffusion-tensor-imaging-based fiber tracking. Neuroimage 30: 1219-1229, 2006

8) Powell JW, Chung CT, Shah HR, Canute GW, Hodge CJ, Bassano DA, Liu L, Mitchell L, Hahn SS: Gamma Knife surgery in the management of radioresistant brain metastases in high-risk patients with melanoma, renal cell carcinoma, and sarcoma. J Neurosurg 109 Suppl: 122-128, 2008

9) Raabe A, Beck J, Rohde S, Berkefeld J, Seifert V: Threedimensional rotational angiography guidance for aneurysm surgery. J Neurosurg 105: 406-411, 2006

10) Rohde V, Spangenberg P, Mayfrank L, Reinges M, Gilsbach JM, Coenen VA: Advanced neuronavigation in skull base tumors and vascular lesions. Minim Invasive Neurosurg 48:
13-18, 2005

11) Stafford SL, Pollock BE, Leavitt JA, Foote RL, Brown PD, Link MJ, Gorman DA, Schomberg PJ: A study on the radiation tolerance of the optic nerves and chiasm after stereotactic radiosurgery. Int J Radiat Oncol Biol Phys 55: 1177-1181, 2003 (review)

12) Tirakotai W, Sure U, Benes L, Krischek B, Bien S, Bertalanffy $\mathrm{H}$ : Image-guided transsylvian, transinsular approach for insular cavernous angiomas. Neurosurgery 53: 1299-1305, 2003

13) Villavicencio AT, Leveque JC, Bulsara KR, Friedman AH, Gray L: Three-dimensional computed tomographic cranial base measurements for improvement of surgical approaches to the petrous carotid artery and apex regions. Neurosurgery 49: $342-353,2001$

Address reprint requests to: Keiji Shimizu, M.D., Department of Neurosurgery, Kochi Medical School, Kochi University, Kohasu, Okoh-cho, Nankoku, Kochi 783-8505, Japan. e-mail:kshimizu@kochi-u.ac.jp 